(2) Open Access Full Text Article

\title{
Patterns of health care utilization for low back pain
}

This article was published in the following Dove Press journal:

Journal of Pain Research

12 August 2015

Number of times this article has been viewed

\author{
Walter F Stewart' \\ Xiaowei Yan' \\ Joseph A Boscarino' \\ Daniel D Maeng' \\ Jack Mardekian ${ }^{2}$ \\ Robert J Sanchez ${ }^{3}$ \\ Michael R Von Korff ${ }^{4}$ \\ 'Geisinger Center for Health \\ Research, ${ }^{2}$ Pfizer, Inc., ${ }^{3}$ Regeneron \\ Pharmaceuticals, Inc., ${ }^{4}$ Group Health \\ Research Institute, Seattle, WA, USA
}

Correspondence: Daniel D Maeng

Geisinger Center for Health Research,

100 North Academy Avenue, MC 44-00,

Danville, PA 17822, USA

$\mathrm{Tel}+\mathrm{I} 5702141688$

Email ddmaeng@geisinger.edu
Background: The purpose of this study was to determine if primary care patients with low back pain (LBP) cluster into definable care utilization subgroups that can be explained by patient and provider characteristics.

Materials and methods: Adult primary care patients with an incident LBP encounter were identified from Geisinger Clinic electronic health records over 5 years. Two-thirds of the cohort had only one to two encounters. Principal component analysis was applied to the data from the remaining one-third on use of ambulatory, inpatient, emergency department, and surgery care and use of magnetic resonance imaging, injections, and opioids in 12 months following the incident encounter. Groups were compared on demographics, health behaviors, chronic and symptomatic disease burden, and a measure of physician efficiency.

Results: Six factors with eigenvalues $>1.5$ explained $71 \%$ of the utilization variance. Patient subgroups were defined as: 1-2 LBP encounters; 2+ surgeries; one surgery; specialty care without primary care; $3+$ opioid prescriptions; laboratory dominant care; and others. The surgery and $3+$ opioid subgroups, while accounting for only $10.4 \%$ of the cohort, had used disproportionately more magnetic resonance imaging, emergency department, inpatient, and injectable resources. The specialty care subgroup was characterized by heavy use of inpatient care and the lowest use of injectables. Anxiety disorder and depression were not more prevalent among the surgery patients than in the others. Surgery patients had features in common with specialty care patients, but were older, had higher prevalence of Fibromyalgia, and were associated primary care physicians with worse efficiency scores.

Conclusion: LBP care utilization is highly variable and concentrated in small subgroups using disproportionate amounts of potentially avoidable care that reflect both patient and provider characteristics.

Keywords: low back pain, primary care, opioids, back surgery, provider efficiency

\section{Introduction}

Low back pain (LBP) is the most common pain problem in the general population ${ }^{1-6}$ and is one of the most common reasons for seeking health care and for use of avoidable care. ${ }^{7,8}$ Guidelines recommend watchful waiting, maintaining activities, and use of nonsteroidal anti-inflammatory drugs, among other conservative actions. ${ }^{9}$ Avoidable care generally occurs because of overdiagnosis, which leads to "subsequent overtreatment, diagnostic creep, shifting thresholds, and disease mongering, processes that ultimately reclassify a healthy low risk patient with mild to moderate problems as a sick patient". ${ }^{10}$ Overdiagnosis manifests as inappropriate use of imaging, treatments (ie, multiple infusions and long-term opioid prescription use), surgery, and other care ${ }^{9,11-15}$ that is also 
related to pain severity, persistence, and recurrence, depression, female sex, neuropathic pain, and narcotics use..$^{4-6,12,13,16}$ A minority of LBP patients account for a dominant share of overall medical care costs, but little is known about how the use of this care unfolds. ${ }^{17-23}$

While considerable research has focused on describing overall utilization of care and predicting costs, relatively little is known about how this care is used for LBP after an initial diagnosis. In this study, we used electronic health record (EHR) data on primary care patients in the 12-month period following an incident LBP encounter to understand how care was used. We first determined how patients clustered by their care utilization patterns. We then examined how care was used within defined patient clusters or groups and then compared these groups on demographics, health behaviors, disease burden, and physician ordering practices.

\section{Materials and methods}

This study involved a retrospective analysis of utilization of care among primary care patients with at least one ambulatory encounter for LBP. We used EHR, not claims data, because of its potential practical utility. EHR data can be evaluated in real time and used at the point of care to assess and determine the course of LBP care management in a way that claims data cannot be used. Also, claims data heavily reflect coverage decisions and utilization management practices that are often idiosyncratic to a given insurer. EHR data, on the other hand, reflects physician orders and treatment decisions. For the purposes of this study, data were extracted from the Geisinger Clinic EHR for the period from January 1, 2007 to December 20, 2011. In this section, we describe the source and patient inclusion and exclusion criteria.

In this study, we defined potentially avoidable care as high end diagnostics, selected treatments (ie, 3+ injections, or 3-5 to $5+$ opioid prescriptions in a 12-month period), urgent or inpatient care without surgery, and surgeries. We emphasize "potentially avoidable" care, because we are unable to determine appropriateness from our data. Nevertheless, guideline-based evidence does not support the use of these potentially avoidable cares for improving diagnostic accuracy in most cases or for improving patient outcomes. ${ }^{6-9}$ Evidence indicates that some, if not a majority, of these types of encounters are avoidable with more accurate diagnosis and symptom management. ${ }^{10}$

\section{Source of population and data}

The Geisinger Health System (GHS) is an integrated delivery system offering health care services to residents in central and northeastern Pennsylvania. The GHS includes the Geisinger Clinic, a multi-specialty group medical practice with 850 physicians and physician's assistants and more than 200 primary care physicians in 41 community practice sites and two ambulatory surgery centers as of the time of this study. Adult (ie, 18+ years of age) primary care patients were the source population for this study. Data for this study have been extracted from the Geisinger Clinic EHR and include patient demographics, smoking history, use of alcohol, and all relevant clinical encounter information such as vitals, encounter type (for example primary, specialty, or emergency department (ED) visit, inpatient admissions, etc.), International Classification of Diseases, Ninth Revision (ICD-9) diagnosis codes, and orders for prescriptions, laboratory, and procedures for LBP at any Geisinger facilities.

\section{Eligibility, follow-up, and utilization of care} Eligible individuals were $18+$ years of age and assigned to a Geisinger Clinic primary care physician before January 1 , 2007 but at least 12 months before December 20, 2010 (end of follow-up) and had at least 12 months of care with their primary care physician before their first recognized encounter for LBP. Patients were excluded if they had less than 12 months of follow-up, and were censored or excluded if they had a diagnosis of malignant cancer, sickle cell anemia, hemophilia, HIV/AIDS, or end-stage renal disease, an organ transplant, a prior long-term care facility admission, or when pregnant. Eligible patients had to have an incident LBP encounter, defined by an encounter diagnosis for LBP with no LBP diagnosis in the prior 12 months.

Data were extracted on all patient encounters in the 12 months before and the 12 months following the incident LBP encounter. Utilization of health care for LBP was defined by the type of encounter (ie, primary care, specialty care, ED or inpatient encounter without surgery, outpatient surgery, inpatient surgery) and by the orders (ie, prescription for opioids, other prescriptions, laboratory, infusion, magnetic resonance imaging [MRI], other imaging, surgery) that were specific to LBP. Providers are required to document one or more ICD-9 codes for the encounter or for any order. An encounter was defined as specific to LBP if an ICD-9 diagnosis, as defined by Cherkin et $\mathrm{al}^{24}$ and modified by Vogt et al, ${ }^{15}$ was documented in the patient record for the encounter or order. The LBP diagnosis was assigned to one of the following diagnostic subgroups based on the first encounter: group 1, back pain with no neurologic findings; group 2, back pain with neurologic findings; group $3 \mathrm{a}$, congenital 
lumbar spine structural disorders; group 3b, acquired lumbar spine structural disorders; and group 4, acquired lumbar spine structural disorders ${ }^{15,24}$ (see Table S1 for a complete list of ICD-9 codes and categorizations).

We excluded telephone encounters unless it was specifically to order an opioid prescription. Telephone calls are frequently documented for prescription renewals or for questions but do not usually constitute an encounter where care decisions are made. On the other hand, because opioid prescriptions are legally controlled, such prescription renewals were considered an actionable decision point. As such, encounters involving opioid prescriptions were included in the analysis.

\section{Analysis}

Principal component analysis was completed to determine how care encounters and orders within encounters clustered together. Encounter types and orders were represented as individual binary variables for each LBP encounter, where three binary variables were included to represent as many as three serial encounters or orders of each type. For instance, three binary variables (ie, surgery_1, surgery_2, and surgery_3) were created to describe situations in which a patient had received one, two, or three or more surgeries during the 12-month period following their incident LBP encounter. If the patient had no surgery, then this would be represented as the following configuration of the binary variables: surgery_1 $=0$, surgery_ $2=0$, and surgery_ $3=0$. Alternatively, if the patient had three or more surgeries, it would be denoted by the following: surgery_1 $=0$, surgery_ $2=0$, and surgery_ $3=1$. The same coding scheme was applied to primary care, specialty care, emergency room, and inpatient encounters without surgery, non-MRI, and MRI. This method of capturing different configurations of the utilization patterns made it possible for factors to form around patients who had multiple encounters of the same type versus none or only a single encounter for a given type.

Incident LBP patients who only had one or two LBP encounters without an MRI, inpatient or surgery care encounter were excluded from the factor analysis and defined as a separate group for all other analyses. Polychoric correlations were estimated and used in the factor analysis given that a categorical variable format was used. Varimax rotation with Kaiser normalization was used to aid in interpretation. ${ }^{25}$ Six factors emerged with an eigenvalue of 1.5 or greater. These factors were used to define patient groups that were then compared on utilization of care and on demographic, health behavior, clinical, diagnosis, and other measures. Groups were compared on sex, age, body mass index, diagnosis of selected cardiometabolic diseases (eg, diabetes and hypertension), chronic episodic conditions (ie, migraine, gastroesophageal reflux disease, asthma), depression, anxiety disorders, sleep disorders, and fibromyalgia. Presence of these conditions was defined by the appearance of the corresponding ICD-9 codes in EHR at least twice, as either one of the encounter diagnoses, or with medication orders on at least two separate encounters, within 12 months of each other. Groups were also compared on the Charlson Comorbidity Index ${ }^{26-28}$ and on a physician efficiency index (PEI) for LBP care that was derived as:

$$
P E I_{i_{j}}=\left[\left(\sum_{i=1}^{N} \operatorname{Cost}_{i}\right)-\text { Cost }_{i}\right] \div(N-1)
$$

PEI represents the physician efficiency index of care for patient $i$ who was treated by provider $j$ during a calendar year. $\mathrm{N}$ represents the total number of patients (including patient $i$ ) treated by provider $j$ during the same calendar year. Therefore, the PEI captures the average per-year cost of care across all patients treated by provider $j$, except for patient $i$, during the given calendar year. Put differently, the PEI captures how the physician treats all patients under his or her care during the year other than the patient under consideration. A relatively high PEI value is therefore indicative of a physician whose practice pattern involves more expensive types of care in general.

The Mantel-Haenszel chi-square test was used for statistical comparisons of ordinal categorical variables, such as age group, depression, total number of encounters in the year before the first LBP encounter, total opioid prescriptions in the year before the first LBP encounter, and the chi-square test was used for nominal categorical variables. For continuous variables, the non-parametric Kruskal-Wallis tes $\mathrm{t}^{29}$ was used to account for non-normality and test median. We used analysis of covariance to compare six subgroups on prior diagnoses for chronic progressive, symptomatic, and psychiatric diagnoses, utilization of care variables, and other factors. We adjusted for age and sex to account for differences among the groups, using the low utilization care group (ie, 1-2 LBP encounters in 12 months) as the reference group. We used pairwise comparisons to specifically identify the subgroups that accounted for overall differences. ${ }^{30}$ All analyses were conducted using SAS version 9.3 (SAS Institute Inc., Cary, NC, USA). 
Cost of care for LBP-related services was not directly available from the EHR. As a substitute, we implemented a cost imputation method based on the subsample of the LBP patient cohort who had Geisinger Health Plan claims data. ${ }^{31}$ Approximately one-third of the incident LBP patients had a Geisinger Health Plan as their primary payer type. These claims data were extrapolated to the remaining twothirds of the patient population who did not have Geisinger Health Plan coverage. For a more detailed description of this methodology, see the Supplementary materials section.

\section{Results}

From January 1, 2007 to December 20, 2011, there were 306,839 eligible primary care patients with $1,264,766$ person-years of follow-up. Thirty-four percent $(n=104,963)$ of these patients had at least one LBP encounter. Of these, the following were excluded: 37,645 prevalent LBP cases actively using care for LBP at the time of cohort inception (ie, January 1, 2007), 17,900 who met previously noted exclusions, and 15,841 who had less than 12 months of follow-up from their incident LBP encounter. The remaining $10.9 \%(n=33,577)$ met the inclusion criteria as an incident LBP patient for a rate of 37.3/1,000 person-years. In the 12-month period following the incident LBP encounter, there were 27,421 ambulatory LBP visits. Approximately $67 \%$ $(n=22,645)$ of the included patients had only one or two LBP encounters with no MRI, inpatient care, or surgery care. These patients were excluded from the factor analysis and instead were clustered separately as another subgroup.

\section{Factor analysis}

In the varimax rotation with Kaiser normalization, six factors had an eigenvalue of 1.5 or greater (Table 1). These six factors explained $71.2 \%$ of the variance among the variables. The dominant factor (Table 1) represented diverse utilization, with high items weights for specialty care, high negative item weights for primary care, and modest weighting for non-surgery inpatient

Table I Factor loading for LBP-related care among an incident LBP patient cohort* from Geisinger Clinic, 2012

\begin{tabular}{|c|c|c|c|c|c|c|c|}
\hline $\begin{array}{l}\text { Number of each } \\
\text { type of care }\end{array}$ & Variable & $\begin{array}{l}\text { Specialty without } \\
\text { primary care }\end{array}$ & Surgical care & $\begin{array}{l}\text { Laboratory } \\
\text { dominant care }\end{array}$ & $\begin{array}{l}\text { Opioid } \\
\text { prescription }\end{array}$ & $\begin{array}{l}\text { 3+ inpatient } \\
\text { encounters }\end{array}$ & One surgery \\
\hline \multirow[t]{10}{*}{ Only one } & PCP encounter & -0.85 & & & & & \\
\hline & Specialty encounter & 0.85 & & & & & \\
\hline & ED encounter & & & & & & -0.81 \\
\hline & Inpatient encounter & & -0.44 & -0.72 & & & \\
\hline & Surgery & & 0.65 & & & & 0.52 \\
\hline & Non-MR image & & & & & & 0.41 \\
\hline & MR image & & & & & & \\
\hline & Laboratory order & & & 0.76 & & & \\
\hline & Opioid order & & & & 0.93 & & \\
\hline & Injection for pain & & 0.88 & & & & \\
\hline \multirow[t]{10}{*}{ Only two } & PCP encounter & -0.95 & & & & & \\
\hline & Specialty encounter & 0.95 & & & & & \\
\hline & ED encounter & 0.54 & & & & -0.7 & \\
\hline & Inpatient encounter & 0.48 & -0.41 & & & & \\
\hline & Surgery & & 0.77 & & & & \\
\hline & Non-MR image & & 0.42 & & & 0.57 & \\
\hline & MR image & & & & & & \\
\hline & Laboratory order & & & 0.83 & & $0.4 I$ & \\
\hline & Opioid order & & & & 0.97 & & \\
\hline & Injection for pain & & 0.89 & & & & \\
\hline \multirow[t]{9}{*}{ Three or more } & PCP encounter & -0.8 & & & & & \\
\hline & Specialty encounter & 0.95 & & & & & \\
\hline & ED encounter & & & $-0.4 \mathrm{I}$ & & -0.5 & \\
\hline & Inpatient encounter & 0.46 & -0.42 & & & 0.66 & \\
\hline & Surgery & & 0.82 & & & & \\
\hline & Non-MR image & & 0.56 & & & & \\
\hline & MR image & & & & & -0.43 & \\
\hline & Laboratory order & & & 0.79 & & & \\
\hline & Opioid order & & & & 0.97 & & \\
\hline Eigenvalues & & 7.07 & 5.79 & 2.47 & 2.35 & 2 & 1.68 \\
\hline
\end{tabular}

Note: *Excluding patients who only had I-2 LBP encounters without MR imaging, inpatient care, or surgery.

Abbreviations: ED, emergency department; MR, magnetic resonance; PCP, primary care physician; LBP, low back pain. 


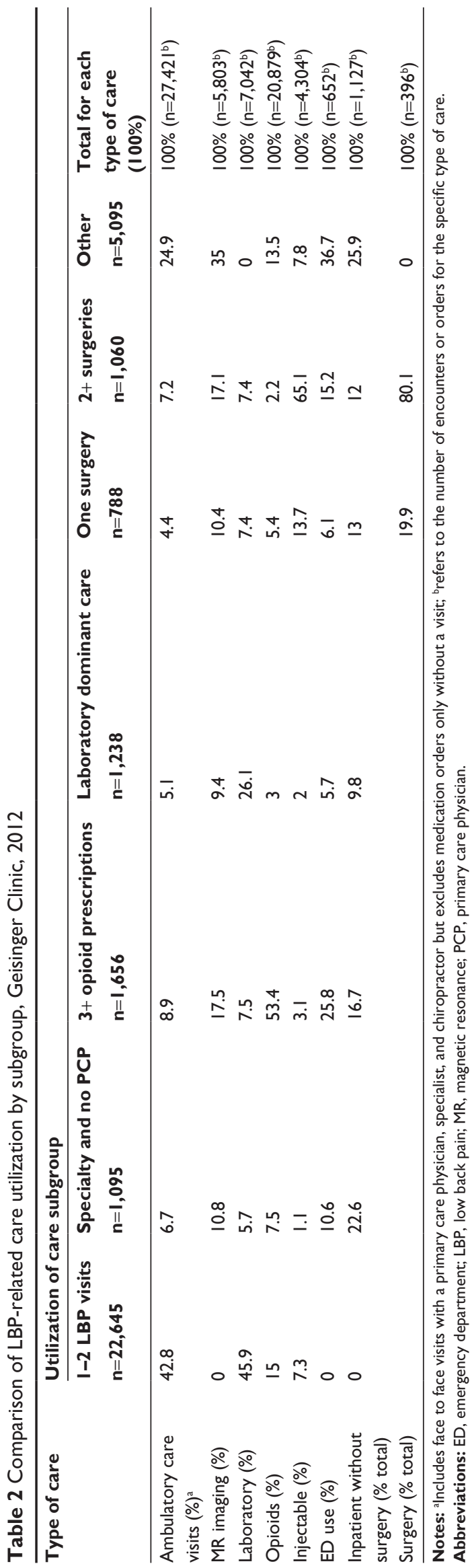

care. Surgical care followed second with loading for the 1,2, and $3+$ surgery groups. However, the group with only a single surgery also loaded separately on the sixth factor. The remaining three factors were represented by item loading for labs with indications for LBP, 3+ opioid prescription orders, and 3+ inpatient encounters. The factors results and other considerations were used to define seven utilization groups as having: 1-2 LBP encounters with no MRI, inpatient encounters, or surgery encounters; $2+$ surgeries; one surgery; at least one specialty care visit without a primary care visits for LBP; $3+$ opioid prescriptions; one or more laboratory orders specific to a LBP indication but with no inpatient care; and patients who did not meet the criteria for the other six groups. The last group included 40 patients with $3+$ inpatient visits, which was too small to form a separate group.

\section{Utilization of care}

Table 2 shows the relative intensity of each type of care used by the seven subgroups. Approximately two-thirds of all ambulatory care was used by the 1-2 LBP encounter subgroup and the "other" subgroups. MRIs were most heavily used by surgery patients as well as by the $3+$ opioid prescription and specialty patients. While these subgroups of patients comprised approximately $14 \%$ of the LBP patients in the sample, they accounted for more than half of all the MRI ordered.

Use of pain control therapies varied. More than half of all opioid prescriptions were prescribed to the $3+$ opioid prescription group. Patients with a single surgery were prescribed considerably more opioids than patients with two or more surgeries. Injectables orders were primarily for patients with $2+$ surgeries $(65.1 \%)$ or a single surgery $(13.7 \%)$. The ED was most heavily used by patients with $3+$ opioid prescriptions (25.8\%) and patients with 2+ surgeries (15.2\%), followed by those with a single surgery or who used specialty care. Inpatient care was most heavily used by the specialty care subgroup and the surgery subgroups, as well as the $3+$ opioid prescription subgroup. The $2.3 \%$ of patients with $2+$ surgeries accounted for $80.1 \%$ of surgeries.

Table 3 compares the proportions of patients within each subgroup who had only one versus two or more of each type of care. The ratio of these two proportions (ie, [\% with only one $] /[\%$ with $2+]$ ) reveals how use of care is concentrated within a smaller number of patients. The lower the ratio, the more utilization of care is concentrated in a smaller number of patients within the defined group. Concentration of care, as indicated by the ratios, varied most for laboratories and ED use. 
Table 3 Comparison of LBP-related care utilization concentration within each subgroup, Geisinger Clinic, 2012

\begin{tabular}{|c|c|c|c|c|c|c|c|c|c|}
\hline \multirow[t]{2}{*}{ Type of care } & \multirow[t]{2}{*}{ Number } & \multicolumn{8}{|c|}{ Utilization of care subgroup } \\
\hline & & $\begin{array}{l}\mathrm{I}-2 \text { LBP } \\
\text { visits } \\
\mathrm{n}=22,645 \\
(67.4 \%)\end{array}$ & $\begin{array}{l}\text { Specialty } \\
\text { and no PCP } \\
n=1,095 \\
(3.3 \%)\end{array}$ & $\begin{array}{l}\text { 3+ opioid } \\
\text { prescriptions } \\
n=1,656 \\
(4.9 \%)\end{array}$ & $\begin{array}{l}\text { Laboratory } \\
\text { dominant care } \\
n=I, 238 \\
(3.7 \%)\end{array}$ & $\begin{array}{l}\text { One surgery } \\
n=788 \\
(2.4 \%)\end{array}$ & $\begin{array}{l}2+\text { surgeries } \\
n=I, 060 \\
(3.2 \%)\end{array}$ & $\begin{array}{l}\text { Other } \\
n=5,095 \\
(15.2 \%)\end{array}$ & $\begin{array}{l}\text { Total } \\
n=33,577 \\
(100 \%)\end{array}$ \\
\hline \multirow[t]{3}{*}{ MR imaging } & I & 0 & 27.6 & 25.4 & 21.6 & 39.5 & 36.8 & 21.4 & 8.3 \\
\hline & $2+$ & 0 & 9.5 & $1 \mathrm{I} .4$ & 6.9 & 13.2 & 17.9 & 6.2 & 2.9 \\
\hline & Ratio* & - & 2.9 & 2.2 & 3.1 & 3.0 & 2.1 & 3.5 & 2.9 \\
\hline \multirow[t]{3}{*}{ Laboratory } & I & 12.2 & 19.1 & 14.4 & 63.5 & 26.2 & 16.4 & 0 & 13.1 \\
\hline & $2+$ & 1.0 & 7.6 & 6.8 & 36.5 & 16.4 & 12.8 & 0 & 3.4 \\
\hline & Ratio* & 12.1 & 2.5 & 2.1 & 1.7 & 1.6 & 1.6 & - & 3.9 \\
\hline \multirow[t]{3}{*}{ Injectable } & $\mathrm{I}-2$ & $\mathrm{I} .4$ & 3.1 & 5.3 & 4.2 & 57.2 & 41.8 & 4.2 & 4.7 \\
\hline & $3+$ & 0 & 0.3 & 0.5 & 0.6 & 2.4 & 49.7 & 0.4 & 1.7 \\
\hline & Ratio* & - & 10.3 & 10.6 & 7.0 & 23.8 & 0.8 & 10.5 & 2.8 \\
\hline \multirow[t]{3}{*}{ ED use } & I & 0 & 4.3 & 3.7 & 2.8 & 4.2 & 4.7 & 3.1 & 1.2 \\
\hline & $2+$ & 0 & 0.4 & 2.3 & 0.1 & 0.4 & 1.3 & 0.7 & 0.3 \\
\hline & Ratio* & - & 10.8 & 1.6 & 28.0 & 10.5 & 3.6 & 4.4 & 4.0 \\
\hline Inpatient but & 1 & 0 & 14.6 & 7.1 & 5.8 & 12.9 & 8.8 & 3.1 & 2.1 \\
\hline \multirow[t]{2}{*}{ not surgery } & $2+$ & 0 & 4.0 & 1.9 & 1.2 & 2.3 & 1.7 & 0.8 & 0.5 \\
\hline & Ratio* & - & 3.7 & 3.7 & 4.8 & 5.6 & 5.2 & 3.9 & 4.2 \\
\hline
\end{tabular}

Notes: *Obtained by dividing the top row $(\mathrm{I})$ by the second row $(2+)$ in each care type category. Values in the table are presented as \%, except for the rows already stated as being measured as a ratio.

Abbreviations: ED, emergency department; LBP, low back pain; MR, magnetic resonance; PCP, primary care physician.

\section{Profile of utilization subgroups}

In the pairwise comparisons (Tables 4 and 5) subgroups differed in one or more two-way comparisons for all variables examined. With only a few exceptions (eg, proportion with a diagnosis of fibromyalgia), the specialty care subgroup did not differ from the reference subgroup (ie, 1-2 LBP encounters in 12 months), but both of these subgroups differed from one or more of the other five subgroups on all variables. In contrast, the $3+$ opioid prescription subgroup was considerably more likely than the reference subgroup to be current cigarette smokers and to have a diagnosis for all other conditions listed in Table 4. The remaining four subgroups were older, but did not have many other features in common. Patients in the laboratory dominant care subgroup were more likely to have hypertension and heart failure and there were substantially higher proportions with chronic episodic disease, severe depression, anxiety and sleep disorders, and fibromyalgia.

Patients with 1-2 LBP encounters had the lowest proportion with a body mass index of $30+\mathrm{kg} / \mathrm{m}^{2}$. Compared with the reference subgroup, both surgery subgroups had high proportions with diabetes and vascular diseases, elevated proportions only for migraine (one surgery group only), arthritis, depression, and sleep disorders, and very high proportions with a diagnosis of fibromyalgia. The "other" subgroup had higher proportions with chronic progressive diseases like the one surgery subgroup and higher proportions with chronic episodic diseases and other disorders like the laboratory dominant subgroup.

In the year before the LBP diagnosis (Table 5), utilization of ambulatory care was in general considerably lower for the 1-2 LBP encounter subgroup and the specialty care subgroup, especially compared with the $3+$ opioid prescription and laboratory dominant subgroups (as shown in the $>15$ category of the rows labeled "Percent by total number of encounters in year before first LBP encounter" in Table 5). The 1-2 LBP encounter and the laboratory dominant subgroups had the highest proportions with a group 1 or 2 LBP diagnosis followed by the $3+$ opioid prescription and other patient subgroups. The specialty care and the 2+ surgery subgroups had the lowest proportion of patients with a group 1 or 2 diagnosis. Finally, the PEI was relatively high for the two surgery subgroups and elevated for patients in the specialty care subgroup.

\section{Cost of LBP care}

The average estimated cost of care per patient for the first 12 months from the incident LBP encounter was $\$ 2,380$. The $2+$ surgeries subgroup accounted for $40.4 \%$ of the total cost of LBP care as represented in the EHR, while the one surgery subgroup accounted for another $19.2 \%$. For the $2+$ surgery subgroup, the median 1-year cost was $\$ 39,504$ 


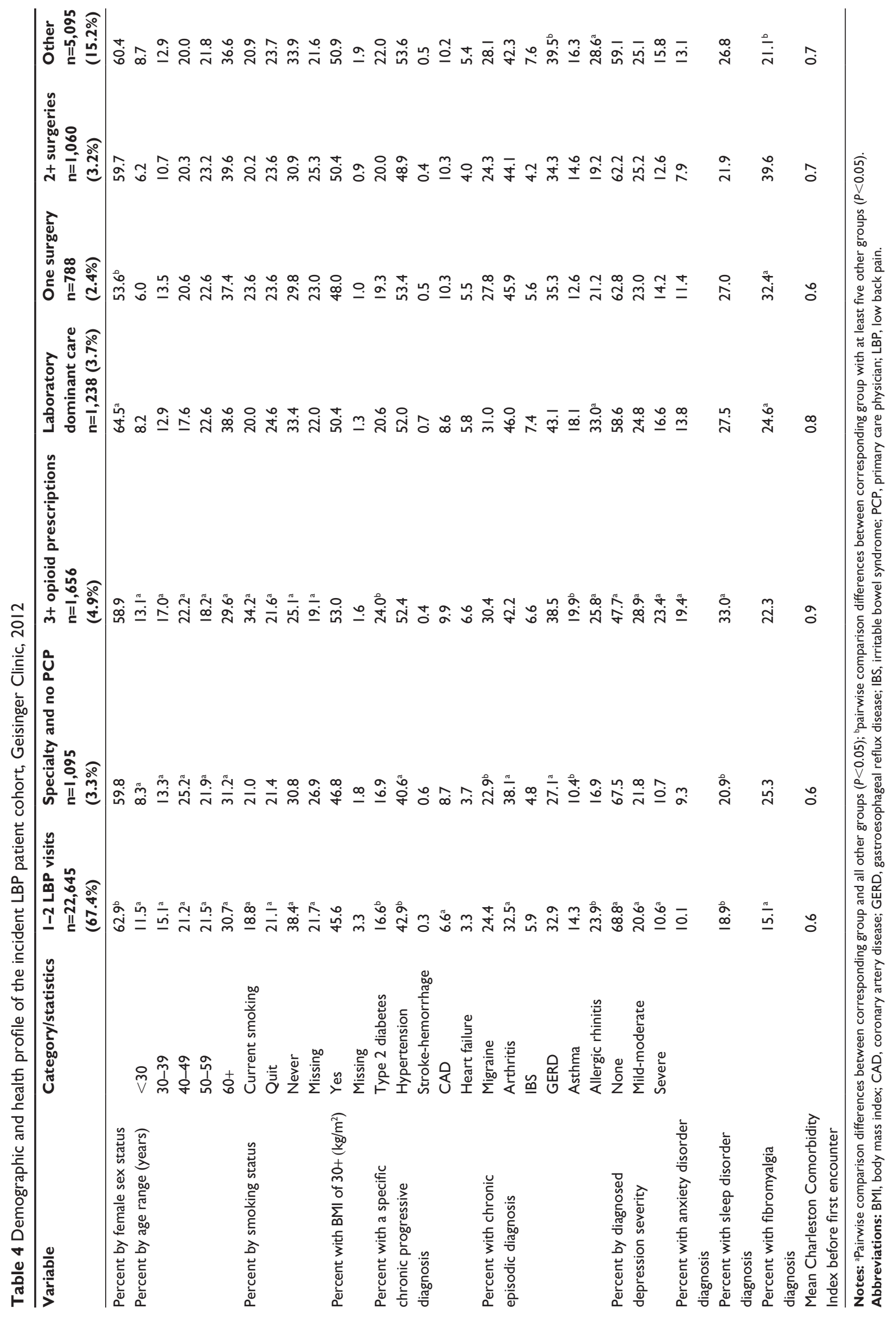




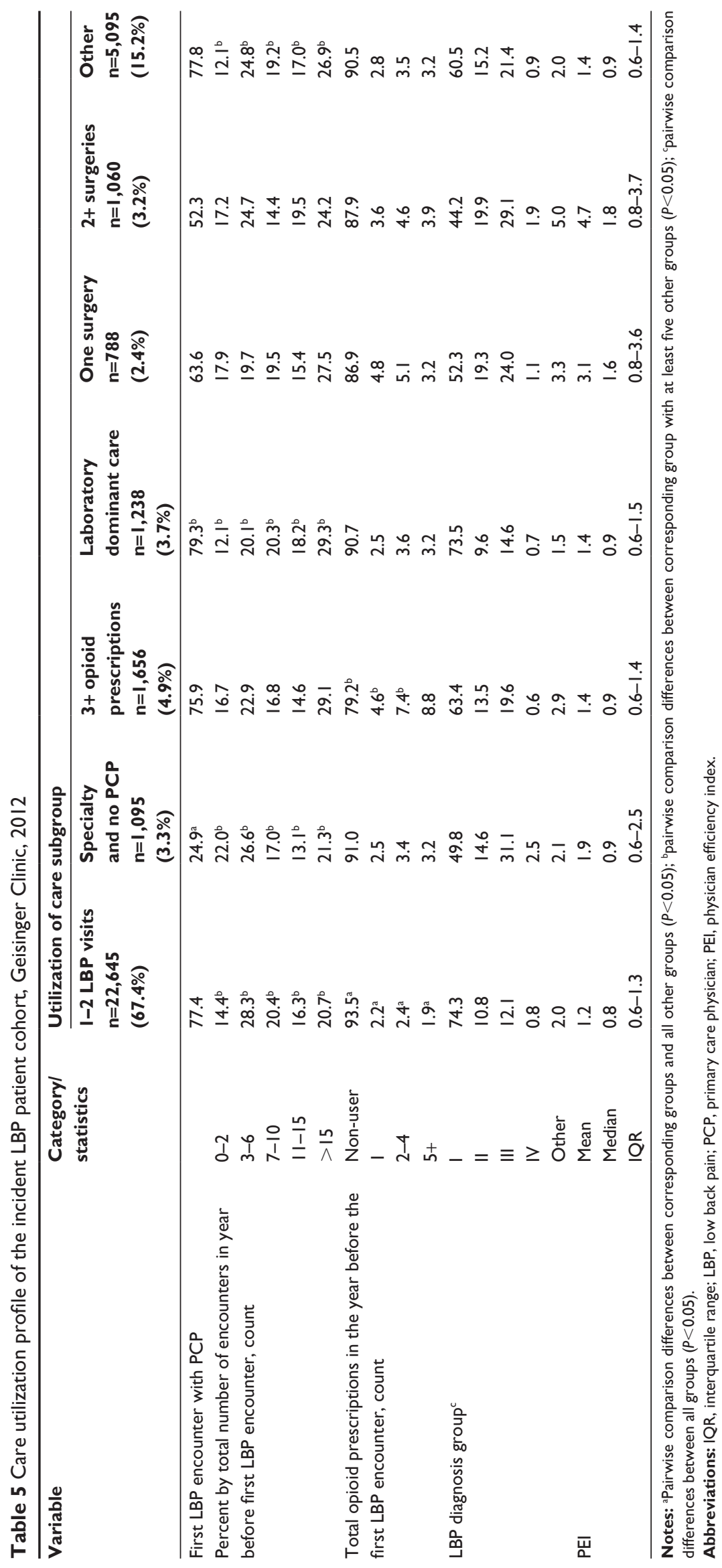


(mean $\$ 47,542$ ) with an interquartile range of $\$ 30,497$ and $\$ 76,080$. This suggests that this $2+$ surgery group is the main drivers of the total cost of LBP care incurred by this cohort.

\section{Discussion}

Two-thirds of LBP patients have only one or two encounters during an incident LBP episode and use very little care. The remaining one-third of patients has very heterogeneous patterns of utilization that appear to fall into six defined groups, four of which account for $13.8 \%$ of patients and most of the MRI, injectable, ED, and inpatient care, with a heavy concentration of utilization in surgery patients. However, use of potentially avoidable care was pervasive across groups. Physician ordering practices and a number of patient health factors differed among the LBP patient groups.

Patients who had $2+$ surgeries had a somewhat unique profile. As expected, these patients were less likely to have benign diagnoses. However, this alone does not explain the use of surgery; $64.1 \%$ had an initial diagnosis that fell into group 1 or group 2 diagnosis as defined by Vogt et al, ${ }^{15}$ a proportion that is similar to that in the specialty care subgroup. In contrast, $71.6 \%$ of patients with a single surgery had a group 1 or 2 diagnosis. Fibromyalgia diagnosis was considerably more common among the $2+$ surgery subgroup followed by the one surgery group, but did not differ significantly across the 1-4 LBP diagnostic groups.

We explored EHR data to identify potential reasons to explain differences among the LBP patient groups. We used the proportion with diagnoses of chronic episodic conditions as an indicator of susceptibility to symptomatic diseases, including polysymptomatic disorders. Relative to other patient subgroups, surgery patients did not have a high burden of these symptomatic conditions, including depression, anxiety disorders, or sleep disorders. In general, anxiety disorder diagnoses were relatively low among the surgery patients. However, the proportion of surgery patients with diagnoses of depression and sleep disorders was consistently higher than that of patients in the specialty care subgroup.

Previous studies have indicated that having a depression or anxiety disorder is more common among LBP patients who are heavy utilizers of care. While the findings from our study are generally consistent with this finding, the elevated prevalence (ie, compared with the 1-2 LBP encounter subgroup) of a diagnosis of depression or anxiety disorder seems largely confined to patients in the $3+$ opioid prescription and laboratory dominant care subgroups, not the specialty care or surgery subgroups. It is possible that the difference between our findings and those of previous studies is that we relied on a physician diagnosis of these conditions versus a direct assessment of depression and anxiety by questionnaire.

The specialty care subgroup appears similar to the surgery subgroups in the use of MRI, ED, and inpatient care and contained the heaviest users of inpatient care. However, this subgroup does not seem to contain heavy users of injectables in general, and certainly not when compared with those with surgery. The specialty care subgroup may be averse to these types of intrusive interventions, including surgery. Alternative explanations include a lower susceptibility to symptomatic conditions or younger age. These patients may simply have had fewer repeat incident LBP episodes (ie, more than a year between the last LBP encounter and a subsequent encounter) which explains, in part, why they did not have surgery.

Table 5 suggests that surgery patients had primary care physicians with the highest (ie, worst) efficiency index, by far, when compared with other patient groups. This score indicates that physician practice explains, in part, the differences in use of care for these two patient groups. Historically, efficiency scores have largely been confined to insurance companies $^{32}$ that derive such measures from claims data to identify providers who are outliers in the volume of care they either provide or order. The rapid adoption of EHRs opens opportunities to derive such scores in real time and to use these scores as decision support aids in the clinical practice setting, a capability that could serve to foster accountable care relationships between providers and payers.

Approximately $5 \%$ of the LBP patients have been defined as moderate to heavy users of opioid prescription medication. Use of opioids has been previously reported to be associated with a higher prevalence of comorbidities and anxiety disorders. ${ }^{33}$ This finding is consistent with our study, as this subgroup of patients consistently contained higher proportions with symptomatic disorder diagnoses and had the highest proportion with severe depression, anxiety disorders, and sleep disorders. This same subgroup also appears to have the highest proportion of current smokers and the heaviest use of opioids in the year before the LBP diagnosis. However, the results suggest that use of opioids was not common among patients with multiple surgeries but was elevated among patients with one surgery. Our findings indicate that these previously identified patient features may be associated with utilization of care, but not necessarily with patients who eventually have LBP surgery.

It is well known that patients with unexplained somatic complaints are relatively high utilizers of health care..$^{34,35}$ 
In our study, symptomatic disorders appeared to be more common among the $3+$ opioid prescription and the laboratory dominant subgroup. However, these two subgroups also have the highest proportion of patients with diagnoses of severe depression and anxiety and sleep disorders. Further, the heavy use of care in these subgroups appears to be selective and primarily confined to the $3+$ opioid prescription group heavily using MRI and ED care when compared with other patient subgroups. The combination of psychiatric diagnoses and diagnoses of other symptomatic conditions including benign LBP, and heavy use of care, in general, may signal that the patient has unmet needs that are not expressed during an encounter or not understood. ${ }^{36}$

There are several potential limitations to our study findings. First, our notion of "incident LBP encounter" is that the sample is comprised of patients who had their first ever LBP encounter and patients with a history of LBP who were experiencing a recurrent episode after a long quiescent period. Identification of a true incident LBP encounter cohort would be helpful in understanding how use of LBP care emerges among first time users versus patients with repeated episodes of LBP over longer periods of time. Second, EHR data offer an incomplete profile of total use of care, in general, and for LBP care. While the Geisinger Clinic provides comprehensive care, it is likely that some care was obtained outside of the clinic and was not documented. As a consequence, patients were likely to be systematically misclassified by utilization group, where the most common error is that use of potentially avoidable care and LBP surgery was underestimated. Lastly, not all LBP patients will seek care, so our finding is restricted to those patients who had access to care and chose to obtain care from care providers.

\section{Acknowledgment}

This study was sponsored by Pfizer Inc., New York, NY, USA.

\section{Author contributions}

WFS, DDM, XY, JAB, and MRVK were responsible for conception and study design. XY, WFS, and DDM acquired the data. WFS, DDM, XY, JAB, JM, RJS, and MRVK were responsible for the analysis and interpretation of the data. WFS, DDM, XY, JAB, and JM drafted the paper and DDM, $\mathrm{XY}, \mathrm{JM}, \mathrm{RJS}$, and MRVK critically revised the final version. All authors agree to be accountable for all aspects of the work in ensuring that questions related to the accuracy or integrity of any part of the work are appropriately investigated and resolved.

\section{Disclosure}

Geisinger Health System (GHS) was paid by Pfizer Inc. in connection with development of this paper. RJS was an employee of Pfizer at the time the study was conducted and the paper was initiated. DDM and JAB are employees of GHS. XY and WFS were employees of GHS at the time the study was conducted and were paid in connection with development of this paper. MRVK and WFS were paid consultants to Pfizer Inc., in connection with this study and development of this paper. The authors report no other conflicts of interest in this work.

\section{References}

1. Deyo RA, Mirsa SK, Martin BI. Back pain prevalence and visit rates: estimates from the US national surveys, 2002. Spine. 2006;31: 2724-2727.

2. Ricci JA, Stewart WF, Chee E, Leotta C, Foley K, Hochberg MC. Back pain exacerbations and lost productive time costs in United States workers. Spine. 2006;31:3052-3060.

3. Stewart WF, Ricci JA, Chee E, Morganstein D, Lipton R. Lost productive time cost due to common pain conditions in the US workforce. JAMA. 2003;290:2443-2454.

4. Andersson GB. Epidemiological features of chronic low-back pain. Lancet. 1999;354:581-585.

5. Von Korff M, Saunders K. The course of back pain in primary care. Spine. 1996;21:2833-2837.

6. Waddell G. Low back pain: a twentieth century health care enigma. Spine. 1996;21:2820-2825.

7. Hart LG, Deyo RA, Cherkin DC. Physician office visits for low back pain. Frequency, clinical evaluation and treatment patterns from a US national survey. Spine. 1995;20:11-19.

8. Cypress BK. Characteristics of physician visits for back symptoms: a national perspective. Am J Public Health. 1983;73:389-395.

9. Goertz M, Thorson D, Bonsell J, et al. Institute for Clinical Systems Improvement. Adult Acute and Subacute Low Back Pain. Updated November 2012. https://www.icsi.org/guidelines__more/catalog_guidelines_and_more/catalog_guidelines/ catalog_musculoskeletal_guidelines/low_back_pain/.

10. Moynihan R, Doust J, Henry D. Preventing overdiagnosis: how to stop harming the healthy. BMJ. 2012;344:e3502.

11. Atlas SJ, Deyo RA. Evaluating and managing acute low back pain in the primary care setting. J Gen Intern Med. 2001;16:120-131.

12. Von Korff M, Wagner EH, Dworkin SF, Saunders K. Chronic pain and use of ambulatory health care. Psychosom Med. 1991;53:61-79.

13. Croft PR, MacFarlene G, Papageorgiou AC, Thomas E, Silman AL. Outcomes of low back pain in general practice: a prospective study. BMJ. 1998;316:1356-1359.

14. Dunn KM, Jordan K, Croft P. Characterizing the course of low back pain: a latent class analysis. Am J Epidemiol. 2006;163:754-761.

15. Vogt MT, Kwoh CK, Cope DK, et al. Analgesic usage for low back pain: impact on health care costs and service use. Spine. 2005;30:1075-1081.

16. Atlas SJ, Nardin RA. Evaluation and treatment of low back pain: an evidence-based approach to clinical care. Muscle Nerve. 2003;27:265-284.

17. Becker A, Held H, Redaelli M, et al. Low back pain in primary care: costs of care and prediction of future health care utilization. Spine. 2010;35:1714-1720.

18. Engel CC, Von Korff, M, Katon WJ. Back pain in primary care: predictors of high health-care costs. Pain. 1996;65:197-204.

19. Eriksen J, Sjøgren P, Ekholm O, et al. Health care utilisation among individuals reporting long-term pain: an epidemiological study based on Danish national health surveys. Eur J Pain. 2004;8:517-523. 
20. Luo X, Pietrobon R, Sun X, et al. Estimates and patterns of direct health care expenditures among individuals with back pain in the United States. Spine. 2004;29:79-86.

21. Mehra M, Hill K, Nicholl D, et al. The burden of chronic low back pain with and without a neuropathic component: a healthcare resource use and cost analysis. J Med Econ. 2012;15:1-8.

22. Parthan A, Evans CJ, Le K. Chronic low back pain: epidemiology, economic burden and patient reported outcomes in the USA. Expert Rev Pharmacoecon Outcomes Res. 2006;6:359-369.

23. Rhee YJ, Taitel, MS, Walker DR, et al. Narcotic drug use among patients with lower back pain in employer health plans: a retrospective analysis of risk factors and health care services. Clin Ther. 2007;29:2603-2612.

24. Cherkin DC, Deyo RA, Volinn E, et al. Use of International Classification of Diseases (ICD-9-CM) to identify hospitalizations for mechanical low back problems in administrative databases. Spine. 1992;17:817-825.

25. Pett MA, Lackey NR, Sullivan JJ. Making Sense of Factor Analysis: The Use of Factor Analysis for Instrument Development in Health Care Research. Thousand Oaks, CA, USA: Sage Publications; 2003.

26. Deyo RA, Cherkin DC, Ciol MA. Adapting a clinical comorbidity index for use with ICD-9-CM administrative databases. J Clin Epidemiol. 1992;45:613-619.

27. Sundararajan V, Henderson T, Perry C, et al. New ICD-10 version of the Charlson Comorbidity Index predicted in-hospital mortality. J Clin Epidemiol. 2004;57:1288-1294.
28. Charlson ME, Pompei P, Ales KL, et al. A new method of classifying prognostic comorbidity in longitudinal studies: development and validation. J Chronic Dis. 1987;40:373-383.

29. Kruskal WH, Wallis WA. Use of ranks in one-criterion variance analysis. J Am Stat Assoc. 1952;47:583-621.

30. Kutner MH, Nachtsheim CJ, Neter J, et al. Applied Linear Statistical Models. 5th ed. New York, NY, USA: McGraw-Hill/Irwin; 2005.

31. Maeng DD, Boscarino JA, Stewart WF, et al. PS1-11: A comparison of electronic medical records vs claims data for rheumatoid arthritis patients in a large healthcare system: an exploratory analysis. Clin Med Res. 2014;12:108.

32. Hussey PS, de Vries H, Romley J, et al. A systematic review of health care efficiency measures. Health Serv Res. 2009;44:784-805.

33. Katz JN. Lumbar disc disorders and low-back pain: socioeconomic factors and consequences. J Bone Joint Surg Am. 2006;88 Suppl 2:21-24.

34. Konnopka A, Schaefert R, Heinrich S, et al. Economics of medically unexplained symptoms: a systematic review. Psychother Psychosom. 2012;81:265-275.

35. Gili M, Luciano JV, Serrano MJ, et al. Mental disorders among frequent attenders in primary care: a comparison with routine attenders. J Nerv Ment Dis. 2011;199:744-749.

36. Schneider A, Wartner E, Schumann I, et al. The impact of psychosomatic co-morbidity on discordance with respect to reason for encounter in general practice. J Psychosom Res. 2013;74:82-85. 


\section{Supplementary materials Cost imputation algorithm}

As is typically the case, electronic health record (EHR) data at Geisinger Clinic lack cost information. To circumvent this problem, we developed a regression-based cost imputation method based on Geisinger Health Plan claims data as outlined below:

1. Start by applying the same inclusion and exclusion criteria to the claims data as done for the EHR data to select the eligible patient population from the claims data.
2. Categorize all encounter types in EHR and claims into a set of mutually exclusive major categories. In this study, we use the following major categories: inpatient visit, outpatient visit, emergency department, diagnostic imaging (ie, X-rays, computed tomography, and magnetic resonance imaging), and all prescription drugs. Professional charges, which are typically available as separate claim types, are assumed to have been incurred in every encounter in EHR.

Table SI Low back pain categories and diagnostic codes

\begin{tabular}{|c|c|c|}
\hline Category & ICD-9 diagnosis code & Diagnosis name \\
\hline Group I: back pain with no & 724.2 & Lumbago \\
\hline \multirow[t]{3}{*}{ neurologic findings } & 724.5 & Backache, unspecified \\
\hline & $846.0-846.9$ & Sprains and strains of sacroiliac region \\
\hline & $847.2,3,9$ & Sprains and strains of back (lumbar, sacrum, unspecified) \\
\hline Group 2 back pain with & 721.42 & Spondylogenic compression of lumbar spinal cord \\
\hline \multirow[t]{5}{*}{ neurologic findings } & 721.91 & Spondylosis of unspecified site, with myelopathy \\
\hline & 722.73 & Lumbar disc disorder with myelopathy \\
\hline & 722.80 & Post-laminectomy syndrome of unspecified region \\
\hline & 724.3 & Sciatica \\
\hline & 724.4 & Thoracic or lumbosacral neuritis or radiculitis, unspecified \\
\hline Group 3a: congenital lumbar & 737.1 & Kyphosis (acquired) \\
\hline \multirow[t]{5}{*}{ spine structural disorders } & 737.20 & Lordosis (acquired) (postural) \\
\hline & 737.3 & Kyphoscoliosis and scoliosis \\
\hline & 739.3 & Nonallopathic lesions, lumbar region \\
\hline & 739.4 & Nonallopathic lesions, sacral region \\
\hline & $756.13-756.19$ & Anomalies of spine \\
\hline \multirow[t]{14}{*}{$\begin{array}{l}\text { Group 3b: acquired lumbar } \\
\text { spine structural disorders }\end{array}$} & $721.5-721.90$ & $\begin{array}{l}\text { Kissing spine; ankylosing vertebral hyperostosis; traumatic spondylopathy; other allied } \\
\text { disorders of spine; spondylosis of unspecified site without mention of myelopathy }\end{array}$ \\
\hline & 722.10 & Lumbar intervertebral disc without myelopathy \\
\hline & 722.2 & Displacement of intervertebral disc, site unspecified, without myelopathy \\
\hline & 722.30 & Schmorl's nodes, unspecified region \\
\hline & 722.32 & Lumbar Schmorl's nodes \\
\hline & 722.52 & Degeneration of lumbar or lumbosacral intervertebral disc \\
\hline & 722.6 & Degeneration of intervertebral disc, site unspecified \\
\hline & 722.90 & Other and unspecified disc disorder of unspecified region \\
\hline & 722.93 & Other and unspecified lumbar disc disorder \\
\hline & 724.00 & Spinal stenosis, unspecified region \\
\hline & 724.02 & Lumbar stenosis \\
\hline & 724.09 & Other \\
\hline & 738.4 & Acquired spondylolisthesis \\
\hline & 756.12 & Congenital spondylolisthesis \\
\hline \multirow[t]{10}{*}{ Group 4: other } & 307.89 & Pain disorders related to psychological factors, other \\
\hline & 722.83 & Post-laminectomy syndrome, lumbar \\
\hline & 724.6 & Disorders of sacrum, including lumbosacral joint instability \\
\hline & 724.8 & Other symptoms referable to back \\
\hline & 724.9 & Other unspecified back disorders \\
\hline & 756.10 & Anomaly of spine, unspecified \\
\hline & 805.4 & Closed fracture of lumbar vertebrae without mention of spinal cord injury \\
\hline & 805.6 & Closed fracture of sacrum or coccyx without mention of spinal cord injury \\
\hline & 805.8 & Fracture of vertebral column without mention of spinal cord injury \\
\hline & 996.4 & Mechanical complication of internal orthopedic device, implant, and graft \\
\hline
\end{tabular}

\footnotetext{
Note: Vogt et al'.
}

Abbreviation: ICD-9, International Classification of Diseases, Ninth Revision. 
3. In the claims data, estimate the following multivariate regression model using a generalized linear model with $\log$ link and gamma distribution function:

Mean cost $=\beta_{0}+\beta_{1}($ encounter type $)+\beta_{2}($ Medicare $)$

$$
+\beta_{3}(\text { age })+\beta_{4}(\text { sex })
$$

"Encounter type" denotes a set of binary indicator variables that represents each major encounter type category (eg, inpatient, outpatient, emergency department); "Medicare" is a binary indicator variable that equals 1 if the patient has Medicare coverage and 0 otherwise; "age" is a continuous variable capturing the patient's age at the time of the study; and "sex" captures the patient's sex.

4. Take the beta coefficient estimates obtained in 3) and apply them to similarly structured EHR data to obtain the estimated mean cost in the EHR.

The above method can be modified by introducing interaction effects between the encounter type variables and age or sex, for instance. In our estimates, the results were not sensitive to such alternative specifications. The resulting cost estimates can be interpreted as "imputed cost" under the hypothetical scenario that the patient had been covered by Geisinger Health Plan. The advantage of this cost imputation method is that it is not necessary that those patients who are included in the claims data be also included in the EHR data; as long as the structure of the EHR data can be modified to accommodate the above regression model, the estimated cost can be obtained for that patient. The disadvantage of this method is that its accuracy may depend on the potentially subjective categorization of claim and encounter types.

\section{References}

1. Vogt MT, Kwoh CK, Cope DK, Osial TA, Culyba M, Starz TW. Analgesic usage for low back pain: impact on health care costs and service use. Spine. 2005;30(9):1075-1081.
Journal of Pain Research

\section{Publish your work in this journal}

The Journal of Pain Research is an international, peer-reviewed, open access, online journal that welcomes laboratory and clinical findings in the fields of pain research and the prevention and management of pain. Original research, reviews, symposium reports, hypothesis formation and commentaries are all considered for publication.

\section{Dovepress}

The manuscript management system is completely online and includes a very quick and fair peer-review system, which is all easy to use. Visit http://www.dovepress.com/testimonials.php to read real quotes from published authors. 